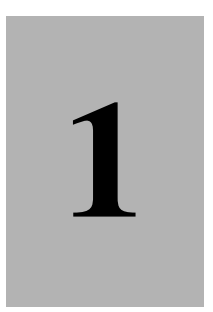

\title{
Flexible Flat Panel Display Technology
}

\section{Gregory P. Crawford}

Division of Engineering, Brown University, Providence RI

\subsection{Introduction}

The manufacturing of flat panel displays is a dynamic and continuously evolving industry. Improvements of flat panel displays are made rapidly as technology improves and new discoveries are made by display scientists and engineers. The cathode ray tube and active matrix liquid crystal display (LCD) recently celebrated their 100th and 25th anniversary, respectively. The arrival of portable electronic devices has put an increasing premium on durable, lightweight and inexpensive display components. In recent years, there has been significant research investment in the development of a flexible display technology. Figure 1.1 shows the evolution away from the bulky CRT display to the thin active matrix LCD for desktop applications, and the much anticipated paper-like flexible flat panel display of the future. To enable a flexible flat panel display, a flexible substrate must be used to replace conventional glass substrates, which can be either plastic or thin glass. Flexible flat panel display technologies offer many potential advantages, such as very thin profiles, lightweight and robust display systems, the ability to flex, curve, conform, roll, and fold a display for extreme portability, high-throughput manufacturing, wearable displays integrated in garments and textiles, and ultimate engineering design freedom (e.g. odd-shaped displays) as shown in Figure 1.2(a). Many of these potential advantages have been the principal 


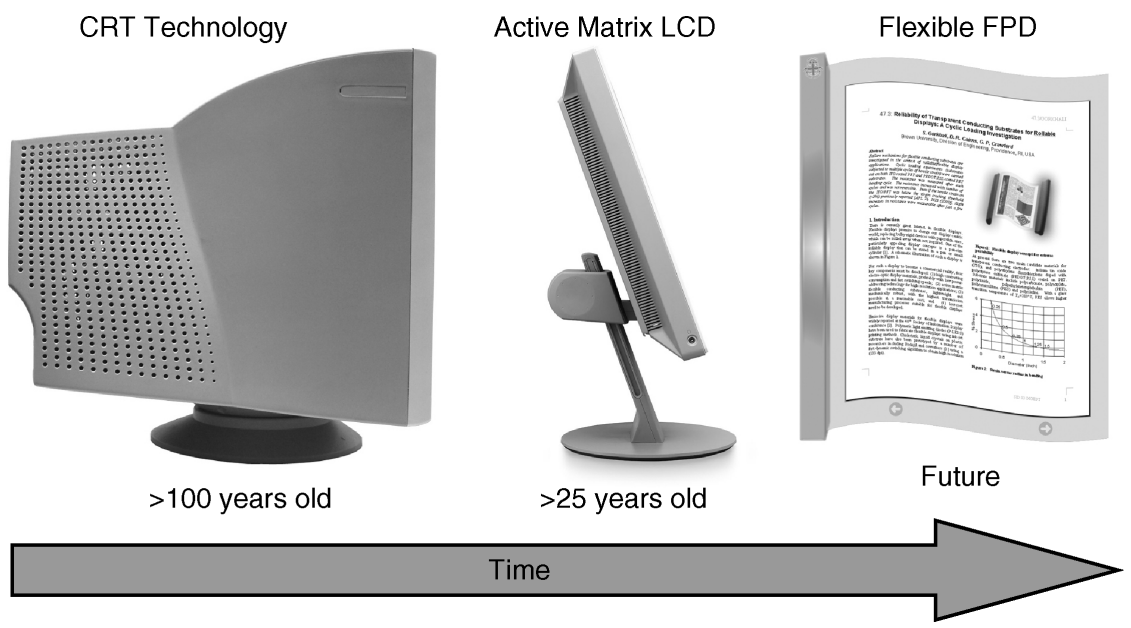

Figure 1.1 Evolution of display technology

driving force behind much of the effort and resources dedicated towards the development of flexible flat panel display configurations.

There are also many new compelling product categories enabled by the promise of plastic display technology. An electronic newspaper, for example, could eventually update headlines throughout the day. If plastic displays on televisions and computers could become

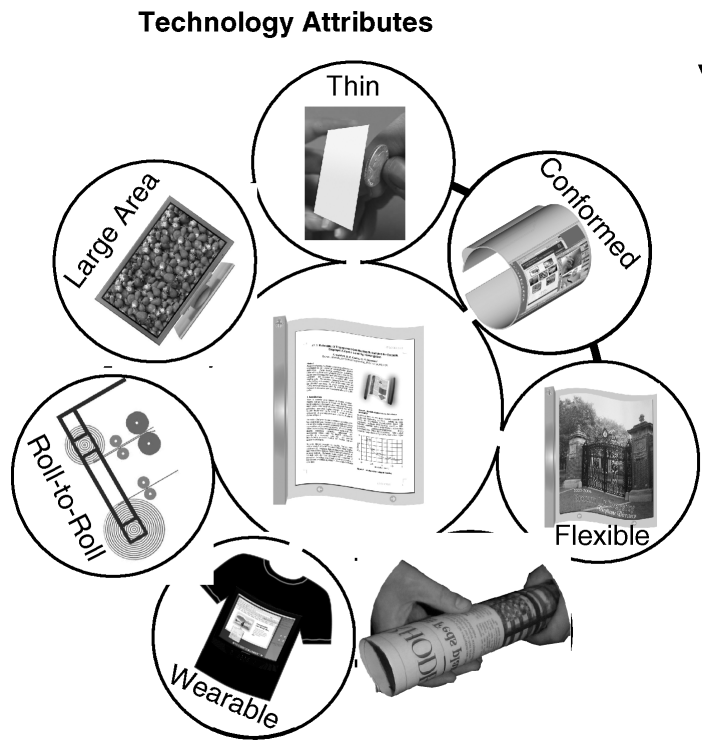

(a)
Technology Convergence

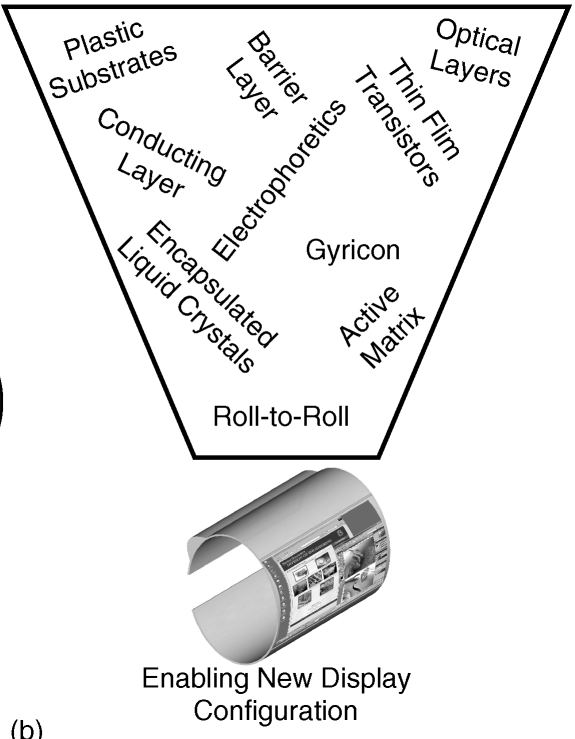

Figure 1.2 (a) The technology attributes of flexible displays and (b) the convergence of the many technologies that are making flexible flat panel displays feasible 
analogous to fabric or paper, they would no longer dominate our physical and aesthetic worlds. We could make them fade from sight when not in service. The television could simply disappear into a painting or tapestry. Your PDA could roll up into a pen that you could stick into your shirt pocket. Instead of adapting our aesthetic sensibilities to incorporate technology into our lives, technology could better reflect our imagination and creativity.

The broad definition of a flexible flat panel display is as follows (Slikkerveer 2003):

A flat panel display constructed of thin (flexible) substrates that can be bent, flexed, conformed, or rolled to a radius of curvature of a few centimeters without losing functionality.

Defining a flexible display is akin to defining modern art (Slikkerveer 2003). Because the diversity of the application space for flexible display technology is so vast, it is hard to propose an all-encompassing definition. The term "flexible display" means different things to different people. Flexible displays may only be flexed once during their lifetime; for example, during manufacturing to create a permanently conformed display. For a rollable display application, however, the display may be rolled and unrolled more than 100 times per day.

The ability to flex a display has fascinated researchers for many years, only today they are being seriously considered for a number of applications and moving closer to the marketplace (Howard 2004; Ong 2004; Kinkade 2004; Hogan 2003; Hellemans 2000; Savage 1999). One of the primary reasons for the increased interest is that many of the necessary enabling technologies for flexible displays are maturing to the extent where reasonablelooking prototypes are being produced by many research and development organizations. As illustrated in Figure 1.2(b), the convergence and evolution of technologies such as flexible substrates, barrier layers, conducting layers, electro-optic materials, optical and functional thin film materials, and thin film transistors (TFTs) is making possible new flexible display concepts.

Flexible display technology can potentially result in many compelling applications not satisfied by a rigid glass-based display. Figure 1.3 shows several artistic renditions of flexible display concepts, such as a large-area, wall-sized reflective screen for use in a conference room setting that could be rolled away when not in use (a), a small portable rollable display (b), an irregular-shaped display used in the steering wheel of an automobile (c), a conformed display integrated in an automobile filling up the entire dashboard (d), a wristband display that is permanently conformed throughout its lifetime (e), and a switchable mask for children, also permanently conformed. Also, there may be a temptation to believe flexible displays will replace glass-based displays for many other applications. While this may be possible at some point in the future, it will be difficult for flexible displays to compete solely on cost alone in the inexpensive and small display module market (e.g. super twisted nematic displays) or in the high-end, high-performance market such as desktop and laptop screens. For the time being, flexible displays will most likely enter the marketplace in a unique way where their positive attributes are clearly capitalized on. The market outlook for flexible displays is surveyed in Chapter 25.

Flexible flat panel display technology constitutes an eclectic research field and potentially large industry in the future. Its highly interdisciplinary range combines basic principles from engineering, physics, chemistry, and manufacturing. The following chapters will provide a comprehensive overview of this exciting and multidisciplinary field. 

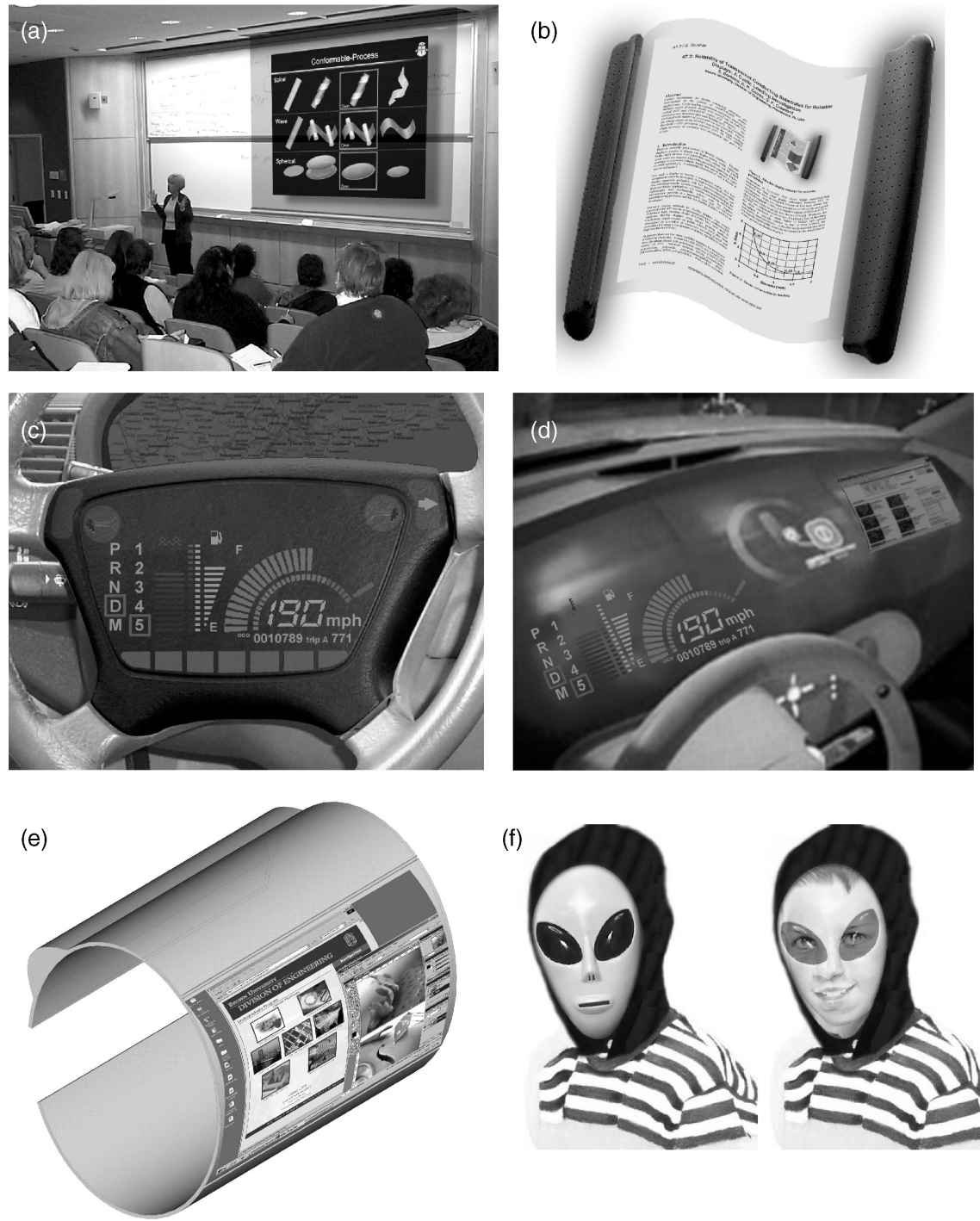

(f)
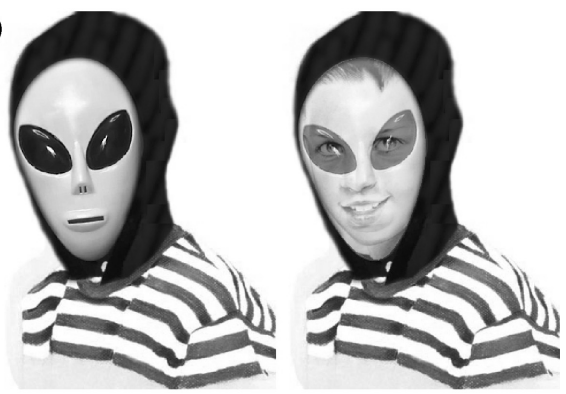

Figure 1.3 Various flexible flat panel display concepts: (a) a direct-view large-area screen; (b) a rollaway display system; (c) an odd-shaped display integrated in a steering wheel of an automobile; (d) a permanently conformed display covering the entire dashboard of an automobile; (e) a permanently conformed display that securely fits around the wrist; and (f) a child's switchable mask. Renditions courtesy of Suraj Gorkhali, Brown University

\subsection{Manufacturing}

Although it may be somewhat of an overstatement, the words "holy grail" are often used to describe the flat panel display community desire to achieve a commercialized flexible display technology (Kincade 2004). One reason why these words are often used is because flexible displays, in principle, are amendable to a roll-to-roll manufacturing process which 


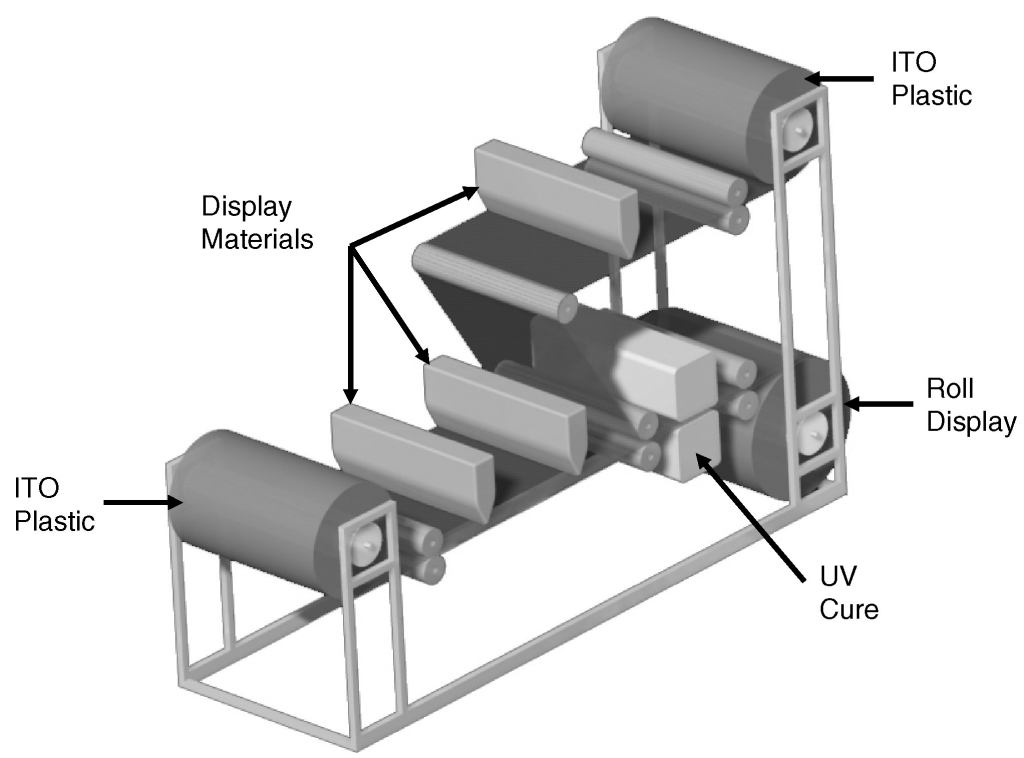

Figure 1.4 A simple schematic diagram of a roll-to-roll manufacturing process

would be a revolutionary change from current batch process manufacturing (Chapter 21). Figure 1.4 shows a simple conceptual illustration of a roll-to-roll manufacturing process where display materials are deposited on indium-tin-oxide (ITO) coated plastic substrates, processed, and rolled back up.

As compared to a batch process, which handles only one component at a time, roll-to-roll processing represents a dramatic deviation from current manufacturing practices. If and when roll-to-roll manufacturing technology matures for display processing, it promises to reduce capital equipment costs, reduce display part costs, significantly increase throughput, and it may potentially eliminate component supply chain issues if all processes are performed with roll-to-roll techniques. Although batch processing can still be employed to manufacture flexible flat panel displays, many researchers and technologists believe that roll-to-roll manufacturing will ultimately be implemented.

\subsection{Enabling Technologies}

The technology of flexible displays includes many components and supporting technologies. Anticipating a new market opportunity, the display industry has been developing display materials targeted specifically at flexible flat panel display requirements. These technologies must be compatible and converge to enable a truly flexible display. The necessary technologies include robust flexible substrates, conducting transparent conducting oxides and/or conducting polymers, electro-optic and reflecting materials, inorganic and organic electronics, and packaging technologies. In addition, many processes must also be developed and optimized in concert with the materials development, such as roll-to-roll manufacturing, 
coating technology, and printing. In reality, these components and processes cannot be optimized independently since a flexible display is a complex system of linked components that must be co-developed in order to function efficiently. It should be made clear that not all technologies described in this book will survive the flexible flat panel race. Since the field is still racing towards commercialization at a rapid pace, it is not at all clear which technologies will win and ultimately become commercialized. The book provides an overview of nearly all the technologies competing in the flexible display landscape, and each topic area provides several solutions for the specific needs of a flexible flat panel display.

\subsubsection{Flexible Substrates}

There are two choices for flexible substrates, which include polymeric and thin glass. Since the flexible substrate represents the fundamental starting component for the display, flexible substrates arguably face the greatest challenges in terms of compatibility with all of the other necessary display layers that need to be integrated onto them. Chapter 2 focuses on polymer films engineered for flexible display technologies. A number of issues are discussed such as process temperature limitations as a function of polymer type, optical properties, thermal properties, and surface smoothness properties. One of the biggest challenges for polymeric substrates is the process temperature required by subsequent display layers (Lueder 2002). It is highly unlikely that flexible displays in the foreseeable future will be completely organic, but rather they will be a hybrid of inorganic and organic layers and components. However, the process temperatures for many inorganic layers have been decreasing (Chapter 5) and the thermal stability of polymer substrates has greatly improved (Chapter 2). This represents one example where technologies are converging in an optimal way to enable flexible displays.

The other solution for flexible substrates is organic based (Chapter 3). Glass has the ultimate barrier properties and is resistant to display process temperature and chemicals, but it lacks the flexibility and ease of handling found in polymeric substrates. Chapter 3 discusses a glass manufacturing process which can process thin glass down to $30 \mu \mathrm{m}$ thicknesses. In order to improve mechanical stability for flexibility and processing, a polymeric layer is deposited on the glass. This hybrid solution enables one to capitalize on the positive attributes of glass, as well as to enable it to be more flexible and process handling friendly.

\subsubsection{Barrier Layers}

When polymeric substrates are employed in flexible display applications, a barrier layer is required to protect the enclosed functional materials and layers from oxygen and water permeation (Chapter 4). Oxygen and water permeation through a flexible substrate is of particular importance to organic light-emitting diode (OLED) devices (Chapter 15). Although single-layer barrier layers do provide the packaged materials with some protection, it appears that multiple layers are necessary for OLED applications for long-term stability. Chapter 4 discloses an inorganic/organic hybrid multilayer solution to create a barrier layer that is beginning to satisfy the demanding requirements of an OLED material. 


\subsubsection{Inorganic Conducting Layers and Mechanical Properties}

Indium tin oxide (ITO) is the typical conducting layer used in display technologies. However, the process temperatures required for ITO on glass to obtain low sheet resistance and high optical throughput properties is incompatible with plastic substrates. Therefore lower-temperature processes have to be developed for ITO in order for it to be considered for flexible display applications (Chapter 5). Although ITO has excellent sheet resistance and optical properties, it does have one shortcoming in the flexible display realm. When ITO is deposited on a polymeric substrate, it can crack (buckle) under tensile (compressive) strain. For a flexible display application, ITO cracking can cause catastrophic failure (Chapter 6). Because of the importance of ITO in display applications, there is significant emphasis on the mechanics of ITO in this book (Chapters 6 and 7). The mechanics of ITO on polymeric substrates is becoming better understood in flexible display applications. In addition, the models and fundamentals learned by studying ITO on polymeric substrates can also be applied to other components, such as inorganic thin film transistors (TFTs) on plastic.

\subsubsection{Organic Conducting Layers and Mechanical Properties}

Conducting polymers are also being considered for flexible display applications (Chapter 8). Although their sheet resistance and optical properties are not as attractive as ITO, they do have exceptional mechanical properties (Chapter 9) and low process temperatures. Chapter 8 describes the fundamentals of the underlying chemistry of conducting polymers and Chapter 9 investigates the mechanics of conducting polymers as compared to ITO. As ITO and conducting polymer technology compete for the conducting substrate solution, there is a new conducting substrate technology based on nanotechnology. Flexible and transparent electrodes have been formed from carbon nanotube dispersions in combination with wet coating processes and printing techniques (Arthur et al. 2004).

\subsubsection{Optical Coatings}

Optical coatings will play an important role in flexible flat panel displays. Many optical films that are used on conventional glass-based displays will be applicable to flexible display configurations. Polarizers, retarders, color filters, antireflection films, and alignment layers for liquid crystals are discussed in Chapter 10. This is an area of research and development that has not been specifically targeted towards the flexible display field, but it does constitute a crucial set of elements in certain flexible display configurations. For example, the paintable LCDs presented in Chapter 18 require thin film polarizers. Additionally, when super twisted nematic (STN) displays are used in a flexible configuration, they require thin film polarizers, retarders, color filters, and backlights (Slikkerveer et al. 2004).

\subsubsection{Thin Film Transistors}

For many electro-optic materials, such as OLEDs (Chapter 15), polymer-dispersed liquid crystals (Chapter 16), paintable LCDs (Chapter 18), electrophoretics (Chapter 19) and 
Gyricon materials (Chapter 20), an active matrix backplane will be required for high resolution. Considerable work is being dedicated to developing various processes to print and pattern organic electronics on polymeric substrates (Chapters 11, 12, 13). There is also significant research and development in developing processes for inorganic TFTs on foil (Chapter 14) and polymer substrates (Chapter 24). The failure mechanisms of TFTs on flexible substrates are also critical to the future success of flexible displays, as discussed in Chapter 14. The success of TFTs for plastic substrates to date has been an enabler for flexible flat panel displays and constitutes a very vital component. They have enabled the development of high-resolution prototypes.

\subsubsection{Electro-Optic Materials}

The various types of electro-optic materials for flexible display applications essentially fall into three categories - emissive, reflective, and transmissive - analogous to the categories for glass-based displays. For emissive applications, OLED materials are being developed that can be small molecule or high molecular weight (Chapter 15). In order to have a truly low-power display, a reflection mode of operation will have to be implemented on flexible substrates. Polymer-dispersed liquid crystals (Chapter 16), chiral liquid crystal dispersions (Chapter 17), encapsulated electrophoretics (Chapter 19), and bichromic ball composites (Chapter 20) all operate in the reflective mode. For electronic book and surrogate paper applications, an efficient reflective mode display is critical to eliminate the need for a powerhungry backlight. Chapter 19 discloses a unique process to paint liquid crystals onto a flexible substrate. Although this technique currently uses a transmissive display mode, the process may be applicable to other materials that can operate in reflection.

\subsubsection{Flexible Display Prototypes}

The primary reason for the interest in flexible displays also arises from the early success of prototypes manufactured by a number of organizations. A number of display system papers are also presented, including a high-resolution LCD based on amorphous silicon (Chapter 22), a full-color LCD based on low-temperature polysilicon (Chapter 23), and an OLED display that was manufactured using a TFT transfer process (Chapter 24). Clearly the ability to prototype high-quality flexible display prototypes has had a tremendous impact on the continued interest and investment in the flexible display arena. The prototypes show the industry what is possible.

\subsubsection{Markets}

Although the future is difficult to predict, the book culminates with a chapter dedicated to the market analysis for flexible flat panel displays. There are many applications in which flexible flat panel displays are suitable for today, but we still do not know what clever applications the industry will come up with once flexible flat panel displays enter the marketplace and how the early adopters react to the new technology. 


\subsection{Conclusions}

The recent development of many components and supporting technologies for flexible flat panel display applications, such as substrates, conducting layers, barrier layers, electro-optic materials, thin film transistor technologies, and manufacturing processes, is accelerating the flexible flat panel display concept towards the marketplace. Very impressive flexible flat panel display prototypes have been manufactured by several display groups around the world, which continues to drive interest and development in the field. The fundamental purpose of the book is to provide a comprehensive work that covers the knowledge needed to understand the various technologies associated with the burgeoning field of flexible flat panel displays and to disclose the state of the art in the field. The book is all-encompassing, with chapters focused on the fundamentals and applications of flexible flat panel displays written by active researchers in their respective fields. The authors come from industry and academia to ensure a well-balanced treatment of the subject matter, which covers fundamental science, engineering, and the state of the art in flexible display technology.

\section{References}

Authur, D., Glatkowski, P., Wallis, P. and Trottier, M. (2004) Flexible transparent circuits from carbon nanotbues. SID Digest of Technical Papers XXXV, 582-585.

Hellemans. A. (2000) Polymer matrix augurs flexible displays. IEEE Spectrum 37, 18-21.

Hogan, H. (2003) Never too thin. Photonics Spectra 37, 66-72.

Howard, W. E. (2004) Better displays with organic films. Scientific American, 76-81.

Kincade, K. (2004) Flexible displays open new windows of opportunity. Laser Focus World 40, 65-69.

Lueder, E. (2002) Plastic substrates for flat panel displays. Proceedings of the 7th Asian Symposium on Information Display, 13-14.

Ong, B. (2004) Flat-panel displays - semiconductor ink advances flexible displays. Laser Focus World 40, 85-88.

Savage, N. (1999) Flexible displays - electronic paper coming to market. Laser Focus World 35, 42-46.

Slikkerveer, P. J. (2003) Bending the rules. Information Display 3, 20-24.

Slikkerveer, P., Bouten, P., Cirkel, P., de Goede J., Jagt, H., Kooyman, N., Nisato, G., van Rijswijk, R. and Duineveld (2004) A fully flexible color display. SID Digest of Technical Papers XXXV, 770-774. 
